\title{
Conditions techniques de raccordement au réseau public BT ou HTA des installations de production autonome d'énergie électrique
}

\author{
Technical conditions for connecting autonomous power stations \\ to the French grid \\ par C. Schmerber \\ Sogeco Hydroénergie
}

Technical rules for connecting a private power station to the french grid with a voltage level up to $50 \mathrm{kV}$ were defined. These rules are applicable for a new power station since mai 1995.

\section{INTRODUCTION}

Les conditions techniques exposées ci-dessous ont pour objet de limiter les perturbations provoquées par les installations de production dans l'exploitation du réseau auquel elles sont raccordées.

\section{TENSION DE RACCORDEMENT, INTEN- SITÉ MAXIMALE ET IMPÉDANCE DES LIGNES ÉLECTRIQUES}

\subsection{Tension de raccordement}

La tension de raccordement est déterminée en fonction de la puissance de l'installation de production autonome selon les correspondances indiquées dans le tableau 1.

\subsection{Intensité maximale et impédance des lignes élec-} triques

Les valeurs indiquées dans le tableau 2 sont des valeurs usuelles qui peuvent être éventuellement modifiées dans le cas de conditions de pose particulières ou en fonction des hypothèses adoptées pour le dimensionnement de la ligne considérée.
Dans les situations de charge du réseau correspondant aux périodes de fonctionnement de l'installation de production, les intensités en ligne doivent être inférieures ou égales aux limites indiquées en 3ème colonne.

2.3 Puissance maximale théoriquement raccordable (calculée avec des Pcc en HTB de 5000 MVA en $225 \mathrm{kV}, 650 \mathrm{MVA}$ en $90 \mathrm{kV}$ et $500 \mathrm{MVA}$ en $60 \mathrm{kV}$ )

Les puissances maximales raccordables sont indiquées dans les tableaux 3 et 4 .

\section{MODIFICATIONS DE LA TENSION DU RÉSEAU DU FAIT D'UNE INSTALLATION DE PRODUCTION}

\subsection{Fluctuations rapides}

L'à-coup de tension lors du couplage, sur le départ, ne doit pas dépasser $5 \%$ pendant plus de $0,5 \mathrm{~s}$ et ne doit pas faire déclencher le disjoncteur de départ au poste source.

Dans le cas des machines synchrones le couplage doit se faire au synchronisme avec des écarts maximaux de $10 \%$ en tension, $0,1 \mathrm{~Hz}$ en fréquence et $10^{\circ}$ en phase.

Dans le cas de génératrices asynchrones, il faut limiter le

Tableau 1: Correspondances puissance - tensions de raccordement.

\begin{tabular}{|c|c|c|}
\hline $\begin{array}{c}\text { Puissance maximale délivrée } \\
\text { sur le réseau }\end{array}$ & Tension de raccordement & Seuils de tension \\
\hline $\mathrm{P} \leq 250 \mathrm{kVA}$ & BT ou HTA & $50 \leq \mathrm{BT}<1000$ \\
\hline $\mathrm{P}<10 \mathrm{MW}$ & HTA & $1000 \leq$ HTA $<50000$ \\
\hline $10 \mathrm{MW} \leq \mathrm{P}<40 \mathrm{MW}$ & HTB $<225 \mathrm{kV}$ & $50000 \leq$ HTB $<130000$ \\
\hline $\mathrm{P} \leq 40 \mathrm{MW}$ & HTB $225 \mathrm{kV}$ & $130000 \leq$ HTB $225<350000$ \\
\hline
\end{tabular}




\begin{tabular}{|c|c|c|c|c|}
\hline Nature & $\begin{array}{c}\text { Section } \\
(\mathrm{mm} \leq)\end{array}$ & $\begin{array}{l}\text { Intensité max. } \\
\text { permanente (A) }\end{array}$ & $\begin{array}{l}\text { Résistance linéique } \\
(0 \mathrm{hms} / \mathrm{km})\end{array}$ & $\begin{array}{l}\text { Réactance linéique } \\
(\mathrm{Ohms} / \mathrm{km})\end{array}$ \\
\hline \multirow{4}{*}{$\begin{array}{l}\text { Lignes nues } \\
\text { Aériennes } \\
\text { Cuivre } \\
\text { Basse tension }\end{array}$} & 22 & 152 & 0,83 & 0,35 \\
\hline & 29,3 & 182 & 0,63 & 0,35 \\
\hline & 38,2 & 213 & 0,486 & 0,35 \\
\hline & 48,3 & 243 & 0,384 & 0,35 \\
\hline \multirow{3}{*}{$\begin{array}{c}\text { Faisceaux torsadés } \\
\text { Aluminium } \\
\text { Basse tension }\end{array}$} & 50 & 205 & 0,641 & 0,1 \\
\hline & 70 & 240 & 0,50 & 0,1 \\
\hline & 150 & 375 & 0,206 & 0,1 \\
\hline \multirow{5}{*}{$\begin{array}{c}\text { Câbles souterrains } \\
\text { aluminium } \\
\text { Basse tension }\end{array}$} & & (hiver) & & \\
\hline & 50 & 205 & 0,641 & 0,1 \\
\hline & 95 & 290 & 0,320 & 0,1 \\
\hline & 150 & 375 & 0,206 & 0,1 \\
\hline & 240 & 485 & 0,125 & 0,1 \\
\hline \multirow{7}{*}{$\begin{array}{l}\text { Lignes nues } \\
\text { Aériennes } \\
\text { Almelec } \\
\text { Moyenne tension }\end{array}$} & 34,4 & 145 & 0,96 & 0,36 \\
\hline & 54,6 & 190 & 0,6 & 0,36 \\
\hline & 75,5 & 240 & 0,44 & 0,36 \\
\hline & 117 & 315 & 0,28 & 0,36 \\
\hline & 148 & 365 & 0,224 & 0,36 \\
\hline & 228 & 480 & 0,146 & 0,36 \\
\hline & 288 & 525 & 0,115 & 0,36 \\
\hline \multirow{7}{*}{$\begin{array}{l}\text { Lignes nues } \\
\text { Aériennes } \\
\text { Alu-acier } \\
\text { Moyenne tension } \\
\text { Phlox }\end{array}$} & 37,7 & 130 & 1,176 & 0,36 \\
\hline & 59,7 & 135 & 0,882 & 0,36 \\
\hline & 75,5 & 175 & 0,697 & 0,36 \\
\hline & 116,2 & 300 & 0,59 & 0,36 \\
\hline & 147,1 & 345 & 0,467 & 0,36 \\
\hline & 228 & 460 & 0,3 & 0,36 \\
\hline & 288 & 525 & 0,238 & 0,36 \\
\hline \multirow[t]{3}{*}{ Pastel } & 147,1 & 345 & 0,279 & 0,36 \\
\hline & 228 & 460 & 0,18 & 0,36 \\
\hline & 288 & 525 & 0,142 & 0,36 \\
\hline \multirow{5}{*}{$\begin{array}{c}\text { Câbles souterrains } \\
\text { aluminium } \\
\text { moyenne tension }\end{array}$} & & (hiver) & & \\
\hline & 50 & 205 & 0,64 & 0,13 \\
\hline & 95 & 290 & 0,32 & 0,13 \\
\hline & 150 & 375 & 0,21 & 0,13 \\
\hline & 240 & 485 & 0,125 & 0,13 \\
\hline
\end{tabular}

Tableau 2 : Intensité maximale et impédance des lignes électriques.

glissement au couplage par une mesure de vitesse ou de fréquence rémanente précise et ou insérer des résistances et coupler en deux temps. La variation de tension doit rester inférieure à $6 \%$.

Dans tous les cas les vitesses de prise de charge ainsi que de cessation de charge ne doivent pas dépasser $4 \mathrm{MW} / \mathrm{mn}$ (indépendamment de tout cas de découplage sur défaut ou de tout autre arrêt d'urgence).

L'amplitude et la fréquence des à-coups et des fluctuations rapides de tension engendrées par l'installation de production au point de livraison doivent être en permanence inférieures ou égales aux valeurs délimitées par la courbe amplitude-fré- quence de la norme CEI 1000-2-2 avec une sévérité de papillotement à court terme (Pst) égal à 1.

\section{- 3.2 Fluctuations lentes}

Pour effectuer les vérifications évoquées dans ce chapitre, les variations de tension peuvent être calculées avec la formule $\Delta \mathrm{U} / \mathrm{U}=(\mathrm{RP}+\mathrm{XQ}) / \mathrm{U} 2$, $\mathrm{P}$ et $\mathrm{Q}$ étant les puissances actives et réactives appelées par les clients (positives) ou fournies par le producteur (négatives), $\mathrm{R}$ et $\mathrm{X}$ les résistance et réactance longitudinales de la ligne ; une élévation de tension correspond à : $\mathrm{DU} / \mathrm{U}<\mathrm{O}$; une diminution correspond à : $\Delta \mathrm{U} / \mathrm{U}>0$. 


\begin{tabular}{|c|c|c|c|c|c|c|c|c|}
\hline \multicolumn{3}{|c|}{ POSTE HTB/HTA } & \multicolumn{6}{|c|}{ PUISSANCE MAXIMALE RACCORDABLE (MVA) } \\
\hline \multirow[t]{3}{*}{$\begin{array}{l}\text { Tension } \\
\text { (kV) }\end{array}$} & \multirow[t]{3}{*}{$\begin{array}{l}\text { Puissance } \\
\text { habituelle } \\
\text { (MVA) }\end{array}$} & \multirow[t]{3}{*}{$\begin{array}{c}\text { Cas } \\
\text { spéciaux }\end{array}$} & \multirow{2}{*}{\multicolumn{2}{|c|}{$\begin{array}{l}\text { Pour ne pas dépasser } \\
\text { la PCC assignée } \\
\text { du matériel HTA }\end{array}$}} & \multicolumn{4}{|c|}{$\begin{array}{l}\text { Sur départ dédié sans utiliser } \\
\text { de protections directionnelles }\end{array}$} \\
\hline & & & & & \multicolumn{2}{|c|}{ Protection du départ } & \multicolumn{2}{|c|}{ Protection du groupe } \\
\hline & & & $\mathrm{Ic}=8 \mathrm{kA}$ & $\mathrm{Ic}=12,5 \mathrm{kA}$ & $\mathrm{A} 0 \mathrm{~km}$ & A $15 \mathrm{~km}$ & $\mathrm{~A} 0 \mathrm{~km}$ & A $15 \mathrm{~km}$ \\
\hline \multirow{6}{*}{$225 / 20$} & 100 & Self 0,7 & - & 40 & 34 & 23 & 34 & 18 \\
\hline & 100 & Self 1,3 & 17 & 65 & 25 & 19 & 25 & 16 \\
\hline & 70 & & - & 10 & 39 & 31 & 39 & 20 \\
\hline & 70 & Self 0,7 & 8 & 55 & 28 & 20 & 28 & 17 \\
\hline & 40 & & 12 & 59 & 25 & 19 & 25 & 16 \\
\hline & $2 \times 40$ & & 12 & 59 & 25 & 19 & 25 & 16 \\
\hline \multirow[t]{6}{*}{$90 / 20$} & 36 & & 25 & 72 & 20 & 16 & 20 & 14 \\
\hline & 30 & & 22 & 70 & 21 & 17 & 21 & 14 \\
\hline & 20 & & 35 & 82 & 17 & 14 & 17 & 12 \\
\hline & 15 & & 45 & 91 & 15 & 12 & 15 & 10 \\
\hline & 10 & & 51 & 98 & 11 & 10 & 11 & 9 \\
\hline & 7,5 & & 53 & 100 & 11 & 9 & 11 & 9 \\
\hline \multirow[t]{5}{*}{$63 / 20$} & 36 & & 30 & 77 & 20 & 16 & 20 & 14 \\
\hline & 30 & & 26 & 74 & 21 & 17 & 21 & 14 \\
\hline & 20 & & 39 & 84 & 17 & 14 & 17 & 12 \\
\hline & 10 & & 54 & 100 & 11 & 10 & 11 & 9 \\
\hline & 5 & & 60 & 105 & 9 & 8 & 9 & 7 \\
\hline \multirow[t]{4}{*}{$225 / 15$} & 100 & Self 0,7 & - & 40 & 26 & 16 & 26 & 12 \\
\hline & 100 & Self 1,3 & 23 & 70 & 18 & 12 & 18 & 10 \\
\hline & 70 & Self 0,7 & 5 & 52 & 22 & 15 & 22 & 11 \\
\hline & 40 & & - & 35 & 25 & 16 & 25 & 11 \\
\hline \multirow[t]{6}{*}{$90 / 15$} & 36 & & 7 & 54 & 20 & 14 & 20 & 10 \\
\hline & 30 & & 6 & 52 & 21 & 14 & 21 & 11 \\
\hline & 20 & & 20 & 65 & 17 & 12 & 17 & 10 \\
\hline & 15 & & 34 & 81 & 15 & 10 & 15 & 8 \\
\hline & 10 & & 40 & 87 & 11 & 9 & 11 & 8 \\
\hline & 7,5 & & 44 & 91 & 11 & 9 & 11 & 7 \\
\hline \multirow[t]{5}{*}{$63 / 15$} & 36 & & 11 & 59 & 20 & 14 & 20 & 10 \\
\hline & 30 & & 12 & 59 & 21 & 14 & 21 & 11 \\
\hline & 20 & & 26 & 73 & 17 & 12 & 17 & 10 \\
\hline & 10 & & 44 & 90 & 11 & 9 & 11 & 8 \\
\hline & 5 & & 53 & 100 & 9 & 7 & 9 & 7 \\
\hline
\end{tabular}

Tableau 3 : Puissance maximale théorique raccordable en fonction des tensions/puissances.

Note : la puissance traversante nest pas prise en compte pour établir ce tableau, elle ne doit pas dépasser la puissance nominale du transformateur HTB/HTA

La charge minimale du réseau est prise égale à un cinquième de la charge maximale du réseau si sa valeur réelle n'est pas connue.

Dans le cas d'un raccordement en basse tension, il faut vérifier que la tension du réseau BT peut être maintenue dans la plage $230 \mathrm{~V}-10 \%$ à $+6 \%$ en tous les points où un client BT est raccordé au même transformateur HTA/BT.

Dans le cas d'un raccordement en HTA, la tension du réseau doit être maintenue dans la plage $-5 \%$ à $+5 \%$ par rapport à la tension contractuelle (en général $20 \mathrm{kV}$ ).

Les centrales dont la puissance installée dépasse 10 MVA doivent être équipées dune régulation de tension agissant dans les limites de réglage de la production et de l'absorption de puissance réactive de celle-ci.

\section{IV - FONCTIONNEMENT DE LA TRANSMIS- SION DES SIGNAUX}

Le producteur ne doit pas perturber le fonctionnement de la transmission des signaux à $175 \mathrm{~Hz}$ ou une autre fréquence proche.

Après étude technique fournie par le distributeur le producteur peut choisir si nécessaire :

- soit d'équiper sa centrale d'un filtre actif série correspondant à la fréquence ou aux fréquences des signaux,

- soit d'équiper sa centrale d'un circuit bouchon passif correspondant à la fréquence ou aux fréquences des signaux, - soit de raccorder sa centrale au poste HTB/HTA sur un jeu de barres HTA spécial " producteur " situé à l'amont des transformateurs d'injection des signaux. 


\subsection{Vérification du fonctionnement de la télécom- mande à $175 \mathrm{~Hz}$}

\subsubsection{Raccordement en basse tension}

$\mathrm{Si}$ la puissance des installations de production à raccorder en ligne au même transformateur HTA/BT ne dépasse pas $40 \%$ de la puissance de ce transformateur, le raccordement est possible. Sinon, les nouvelles installations doivent être raccordées directement à ce transformateur HTA/BT ou à un autre.

\subsubsection{Raccordement en haute tension}

Il n'y a pas lieu de procéder à une vérification de la bonne transmission des signaux de télécommande si la puissance totale des installations raccordées à ce poste est inférieure ou égale à $5 \%$ de la puissance du ou des n-1 transformateurs de plus faible puissance du poste.

Dans tous les autres cas le calcul devra être effectué. On désignera respectivement par $\tau_{\mathrm{am}}$, et $\tau_{\mathrm{av}}$ les taux $175 \mathrm{~Hz}$ amont et aval calculés sur le réseau.

Tous les calculs des taux considérés seront effectués dans la situation suivante :

- la puissance de court-circuit amont correspond à un niveau d'alimentation du poste source en situation peu fréquente (quelques semaines par an) et où l'un des transformateurs H.T.B./H.T.A. du poste source est déconnecté (s'il y en a plus d'un).

- le réseau H.T.A. sera chargé à sa charge maximale $P_{\text {cons }}$ avec une tg $\phi=0,4$. Le réseau H.T.A. sera dans son schéma normal.

- les batteries de condensateurs du poste source seront supposées non connectées.

- l'ensemble des consommateurs sera modélisé par une impédance ramenée au poste source calculée par la formule donnée dans le bulletin Electra n $^{\circ} 77$ :

$\mathrm{Xs}$ en série avec Rs, le tout en parallèle avec Xp

$$
\begin{aligned}
& \mathrm{Rs}=\mathrm{U}^{2} / \mathrm{P} \\
& \mathrm{Xs}=0,073 \times \mathrm{Rs} \times(175 / 50) \\
& \mathrm{Xp}=[(175 / 50) \times \mathrm{Rs}] /(6,7 \times \operatorname{tg} \phi-0,74)
\end{aligned}
$$

- la réactance $\mathrm{Xg}$ des groupes du producteur sera la réactance inverse multiplié par 175/50

- les réactances des lignes aériennes seront prises égales à $0,36 \Omega / \mathrm{km}$ et celles des lignes souterraines à $0,13 \Omega / \mathrm{km}$ (à multiplier par 175/50).

- les résistances des câbles, des transformateurs, des lignes et des groupes seront négligées, leur influence est négligeable ou de second ordre sur les résultats.

- le taux d'injection du $175 \mathrm{~Hz}$ est pris à sa valeur théorique de $2,3 \%$.

Lorsque les taux du signal $175 \mathrm{~Hz}$ avant raccordement sont tels que $\tau_{\mathrm{am}} \leq 0,4 \%$ et $\tau_{\mathrm{av}} \geq 1,4 \%$.

Si les nouveaux taux calculés en prenant en compte l'installation de production autonome respectent les limites suivantes : taux amont $\leq(0,4+0,03) \%$ et Taux aval $\geq(1,4$ $0,03) \%$ alors le raccordement sans dispositif spécifique de protection de la transmission tarifaire sera accepté.

Lorsque les taux du signal $175 \mathrm{~Hz}$ avant raccordement sont tels que $\tau_{\mathrm{am}}>0,4 \%$ et $\tau_{\mathrm{av}}<1,4 \%$, si les nouveaux taux calculés en tenant compte de l'installation de production autonome respectent les limites suivantes : Taux amont $\leq$ $\tau \mathrm{am}+0,03 \%$ et Taux aval $\geq \tau \mathrm{tav}-0,03 \%$, alors le raccordement sans dispositif spécifique de protection de la transmis- sion tarifaire sera accepté.

Sinon, il faut refaire le calcul en intégrant dans la nouvelle installation un circuit bouchon dont les caractéristiques sont données par le producteur au distributeur.

Si les nouveaux taux calculés avec le circuit bouchon respectent les limites précédentes, alors le raccordement avec ce circuit bouchon sera accepté.

Si les nouveaux taux calculés avec le circuit bouchon ne respectent pas ces limites, le raccordement à l'amont des transformateurs d'injection $175 \mathrm{~Hz}$ au poste source ou le filtre actif série seront seuls acceptés.

Dans l'exemple du tableau 5 , le producteur pourra être raccordé sans dispositif spécifique.

Une vérification analogue et les dispositions correspondantes devront être mises en place en temps utile par le producteur pour permettre le bon fonctionnement des émissions tarifaires à $188 \mathrm{~Hz}$, dès leur utilisation sur le réseau local ou lorsque le distributeur utilise une autre fréquence d'émission des signaux que $175 \mathrm{~Hz}$.

Le distributeur fournit au producteur les éléments nécessaires pour modéliser le circuit bouchon, à savoir l'impédance du réseau HTB, l'impédance du transformateur HTB/HTA et l'impédance modélisée du réseau HTA compte tenu de sa charge, à la fréquence $175 \mathrm{~Hz}$.

\section{IV $\square$ FILTRES}

\subsection{Filtre actif série}

\subsubsection{Caractéristiques}

Le filtre actif série est un dispositif qui détecte les signaux arrivant chez le producteur et émet un signal égal en fréquence, amplitude et phase (ou d'un niveau légèrement supérieur en amplitude). Cette contre-injection annule les courants absorbés par l'installation du producteur et permet de maintenir à son niveau initial, ou même d'améliorer, le niveau des signaux.

Il est constitué d'un onduleur de tension et d'un transformateur d'injection raccordé en série entre l'installation de production et son point de raccordement au réseau.

Il est auto-surveillé et consultable à distance.

\subsubsection{Dimensionnement}

Il doit être dimensionné pour l'intensité maximale qui le traverse.

Il doit être prévu pour la tension assignée de $24 \mathrm{kV}$.

Il doit tenir l'intensité maximale de court-circuit du réseau $(12,5 \mathrm{kA})$.

\subsubsection{Découplage}

Aucun découplage ne peut être demandé au producteur sur défaut du signal de transmission si le filtre actif ne présente pas d'anomalie.

En cas de défaut du filtre actif, le distributeur ne peut demander un découplage que s'il constate un niveau anormal du signal tarifaire. Le producteur doit réaliser cette manœuvre dans les plus brefs délais (moins d'une heure).

\subsection{Circuit bouchon passif à $175 \mathrm{~Hz}$ 5.2.1 Caractéristiques}

Les bobines d'inductances doivent être conformes à la norme NF C 52-300, de type sec à noyau d'air. La tolérance sur la valeur nominale de la réactance est de $+/-1 \%$. La variation de la réactance en fonction de la température 
CONDITIONS TECHNIQUES DE RACCORDEMENT AU RÉSEAU PUBLIC BT OU HTA

\begin{tabular}{|c|c|c|c|c|c|c|}
\hline Tension réseau & $U$ & 20 & KV & & & \\
\hline Réseau amont & Pcc & 1500 & MVA & & 0,933 & Ohms \\
\hline \multirow[t]{2}{*}{ Transfo HTB/HTA } & $P$ & 40 & MVA & $X$ amont & = & \\
\hline & Ucc & 17 & $\%$ & $\mathrm{X}$ transfo & 5,950 & Ohms \\
\hline \multirow[t]{5}{*}{ Consommateurs } & $\mathrm{P}$ & 27 & MW & & & \\
\hline & $\operatorname{Tg} \phi$ & 0,4 & & $X$ cons & 7,78 & Ohms \\
\hline & Rs & 14,81 & Ohms & $\mathrm{R}$ cons & 9,20 & Ohms \\
\hline & Xs & 3,79 & Ohms & Rho cons & 12,25 & Ohms \\
\hline & $\mathrm{Xc}$ & 26,73 & Ohms & Theta cons & 40,23 & $\infty$ \\
\hline \multicolumn{7}{|c|}{ PRODUCTEUR } \\
\hline \multirow[t]{2}{*}{ Ligne } & \multicolumn{2}{|c|}{ Réactance 0,36} & $\mathrm{Ohm} / \mathrm{km}$ & & & \\
\hline & Longueur & r $\quad 0,12$ & $\mathrm{Km}$ & XI & 0,0432 & Ohms \\
\hline \multirow[t]{2}{*}{ Transfo groupe } & $P$ & 6 & MVA & & & \\
\hline & Ucc & 6,5 & $\%$ & $\mathrm{X}$ transfo & 15,17 & Ohms \\
\hline Groupe & $P$ & 5 & MW & & & \\
\hline & $\mathrm{Xi}$ & 16 & & $X \mathrm{~g}$ & 44,80 & Ohms \\
\hline Impédance & $\mathrm{Rp}$ & 0 & Ohms & $\mathrm{Xp}$ & 60,01 & Ohms \\
\hline producteur & Rho p & 60,01 & Ohms & Theta $p$ & 90 & $\infty$ \\
\hline & & $\overline{\mathrm{CIR}}$ & JIT BOUCHON & & & \\
\hline & $\mathrm{L}(\mathrm{mH})$ & $\mathrm{R}(\mathrm{Ohm})$ & C (micro-Farad) & $Q$ & $\mathrm{Fa}$ & cord \\
\hline Caractéristiques & 3,6 & 0,08 & 230 & 50 & 174,9061 & $\mathrm{~Hz}$ \\
\hline Self & $\mathrm{RI}$ & 0,08 & Ohms & $\mathrm{XI}$ & 3,958 & Ohms \\
\hline Capacité & Rc & 0 & Ohms & $\mathrm{Xc}$ & 3,954 & Ohms \\
\hline Impédance & Rcb & 194,893 & Ohms & $\mathrm{Xcb}$ & $-14,3$ & Ohms \\
\hline Circuit bouchon & Rho cb & 195,417 & Ohms & Theta $\mathrm{cb}$ & $-4,196$ & $\infty$ \\
\hline & & PRODUCTEL & + CIRCUIT BOI & $\mathrm{CHON}$ & & \\
\hline Impédance Producteur & Rpcb & 194,893 & Ohms & Xpcb & 45,71 & Ohms \\
\hline + Circuit bouchon & Rho pcb & 200,18 & Ohms & Theta pcb & 13,20 & $\infty$ \\
\hline & & X AMONT E & AVAL PRODUCT & EUR SEUL & & \\
\hline Paramètres & & $\mathrm{R}(\mathrm{Ohm})$ & $\mathrm{X}(\mathrm{Ohm})$ & Rho & (hm) & Theta $(\infty)$ \\
\hline Réseau amont & & 0 & 0,93 & & & 90 \\
\hline Transfo HTB/HTA & & 0 & 5,95 & & & 90 \\
\hline Consommateurs & & 9,20 & 7,78 & & & 40,23 \\
\hline Producteur & & 0 & 60,01 & & & 90 \\
\hline Consommateurs + proc & ducteur & 7,08 & 7,85 & & & 47,96 \\
\hline Total sans producteur & . & 9,20 & 14,67 & & & 57,90 \\
\hline Total avec producteur & & 7,08 & 14,73 & & & 64,34 \\
\hline Seuil injection & & U175 & $2,3 \%$ & & & $460 \mathrm{~V}$ \\
\hline & SANS $P$ & ODUCTEUR & & AVEC P & ODUCTEUF & \\
\hline$I(A)$ & 26,57 & Angle & $-57,90$ & 28,14 & Angle & $-64,34$ \\
\hline V175 amont & 24,80 & Angle & 32,10 & 26,27 & Angle & 25,66 \\
\hline V175 aval & 320,16 & Angle & $-17,67$ & 297,45 & Angle & $-16,38$ \\
\hline TAUX AMONT & & 0,12 & & & 0,13 & \\
\hline TAUX AVAL & & 1,6 & & & 1,49 & \\
\hline & AUX AMONT & ET AVAL PR & UCTEUR AVEC & CIRCUIT BO & CHON & \\
\hline Paramètres & & $\mathrm{R}(\mathrm{Ohm})$ & $\mathrm{X}(\mathrm{Ohm})$ & Rho & hm) & Theta $\left({ }^{\circ}\right)$ \\
\hline Réseau amont & & 0 & 0,93 & & & 90 \\
\hline Transfo HTB/HTA & & 0 & 5,95 & & & 90 \\
\hline Consommateurs & & 9,20 & 7,78 & 12 & & 40,23 \\
\hline Producteur & & 194,893 & 45,71 & 200 & & 13,20 \\
\hline Consommateurs + proc & ducteur & 8,92 & 7,15 & & & 38,74 \\
\hline Total sans producteur & & 9,20 & 14,67 & & & 57,90 \\
\hline Total avec producteur & & 8,92 & 14,04 & & & 57,58 \\
\hline Seuil injection & & U175 & $2,3 \%$ & U & & $460 \mathrm{~V}$ \\
\hline
\end{tabular}

Tableau 5 : Exemple de raccordement au réseau. 


\begin{tabular}{|l|l|l|l|l|l|l|}
\hline & \multicolumn{3}{|c|}{ SANS PRODUCTEUR } & \multicolumn{3}{c|}{ AVEC PRODUCTEUR } \\
\hline I (A) & 26,57 & Angle & $-57,90$ & 27,66 & Angle & $-57,58$ \\
\hline V175 amont & 24,80 & Angle & 32,10 & 25,82 & Angle & 32,42 \\
\hline V175 aval & 320,16 & Angle & $-17,67$ & 316,21 & Angle & $-18,83$ \\
\hline TAUX AMONT & \multicolumn{3}{|c|}{$\mathbf{0 , 1 2}$} & \multicolumn{3}{c|}{$\mathbf{0 , 1 3}$} \\
\hline TAUX AVAL & $\mathbf{1 , 6}$ & $\mathbf{1 , 5 8}$ \\
\hline
\end{tabular}

Tableau 5 : Exemple de raccordement au réseau (suite)

ambiante doit être inférieure à $0,2 \%$ pour $20^{\circ} \mathrm{C}$. Le facteur de qualité ( $\mathrm{L} / \mathrm{R}$ pour $175 \mathrm{~Hz}$ ) doit être inférieur à 60 , le courant nominal permanent à $50 \mathrm{~Hz}$ au moins égal à celui de la centrale (plus $10 \%$ environ). La tenue au courant de courtcircuit du réseau doit être assurée (12,5 kA).

Les condensateurs doivent être conformes à la norme NF C 54-102, sans fusibles incorporés. La capacité nominale est définie pour une température de référence de $20^{\circ} \mathrm{C}$ avec une tolérance sur la valeur nominale de la capacité de $+/-4 \%$. La variation de la capacité en fonction de la température ambiante doit être inférieure à $1 \%$ pour $20 \mathrm{~K}$.

La variation de la fréquence de coupure du circuit bouchon doit être de moins de $1 \%$ pour une variation de la température de $40^{\circ} \mathrm{C}$, sinon, le circuit bouchon doit être installé dans un bâtiment tempéré $\left(20^{\circ} \mathrm{C}+/-1,0^{\circ} \mathrm{C}\right.$ environ).

\subsubsection{Vérifications, réglages et contrôle}

Le contrat du producteur doit prévoir que les caractéristiques électriques du circuit et notamment la valeur de la fréquence de coupure, seront vérifiées par des mesures d'impédance à des fréquences convenables, et qu'un réglage sera effectué si cette fréquence sort des limites admissibles, c'est-à-dire ne permet plus de garantir la plage de variation prévue :

- avant la période de production,

- au moins une fois par mois pendant cette période.

La vérification annuelle avant la période de production doit être effectuée par un organisme de contrôle qualifié.

Le contrôle est réalisé au moyen d'un appareil de contrôle de la tension à $175 \mathrm{~Hz}$ au poste source muni d'une alarme réglée sur un seuil minimal.

\subsubsection{Modalités de découplage et de recouplage d'une ins-} tallation de production autonome

Ces modalités seront mises en œuvre pour les installations de production de plus de $1 \mathrm{MW}$ raccordées à un poste source équipé d'un système de surveillance des émissions $175 \mathrm{~Hz}$ permettant les fonctionnalités suivantes :

- mesure à chaque émission du taux aval $175 \mathrm{~Hz}$ en pourcentage de la tension nominale,

- réglage sur un seuil de taux aval $175 \mathrm{~Hz}$ prédéfini.

Lorsque le taux mesuré devient inférieur à ce seuil, une signalisation de franchissement est actionnée.

Ce système de surveillance est destiné à être associé à un émetteur de télécommande afin, lors du franchissement du seuil mentionné, d'envoyer via la liaison spécialisée de télédéclenchement. un ordre de découplage vers les installations de production.

Lors d'une émission $175 \mathrm{~Hz}$, le système de surveillance au poste source comparera le taux aval effectif du signal tarifaire au seuil prédéfini. Si ce taux aval est inférieur à ce seuil, le système de surveillance au poste source enverra par la liaison spécialisée de télédéclenchement l'ordre d'ouver- ture du disjoncteur de couplage de l'installation de production au réseau H.T.A. L'installation de production n'étant plus couplée au réseau H.T.A, le chargé de conduite du réseau H.T.A. renverra une nouvelle émission tarifaire. Si le taux aval ne s'améliore pas, l'autorisation de redémarrage de l'installation de production sera transmise dès que possible au producteur par le chargé de conduite du réseau H.T.A. Une enquête technique sera ensuite menée par les services compétents du distributeur. Si le taux aval retrouve une valeur supérieure au seuil $175 \mathrm{~Hz}$ après le découplage de l'installation de production, cette dernière ne sera autorisée à redémarrer qu'après la vérification et la remise en conformité par le producteur de ses installations (en ce qui concerne leur effet sur le taux aval prévu lors de l'autorisation de leur raccordement au réseau). Si la même séquence (baisse du taux aval au-dessous du seuil $175 \mathrm{~Hz}$ puis taux à nouveau au-dessus du seuil après découplage de l'installation) se reproduit pour la même installation, celle-ci ne sera pas autorisée à redémarrer avant l'enquête technique menée par les services compétents du distributeur ou un organisme qualifié.

Dans tous les cas, la mise en service ou la reprise de service d'une installation de production ne pourra être opérée qu'après autorisation du distributeur qui la transmettra dans les délais les plus courts compatibles avec les contraintes d'exploitation du réseau. La mise ou la remise en service sera alors exécutée par le producteur.

\section{HARMONIQUES ET DÉSÉQUILIBRES}

Les seuils harmoniques doivent être inférieurs aux taux fixés dans le tableau 6 avec un taux global inférieur à $8 \%$ pour les harmoniques de rang 2 à 40 .

La variation du taux de déséquilibre des tensions due au producteur ne devra pas dépasser $2 \%$.

\section{VII — PUISSANCE RÉACTIVE}

L'installation du producteur doit pouvoir fournir une puissance réactive nominale Qn, définie ainsi :

\begin{tabular}{|c|c|}
\hline Puissance active & Puissance réactive \\
\hline $\mathrm{P}<10 \mathrm{MVA}$ & $\mathrm{Qn}=0,5 \mathrm{Sn}$ \\
\hline $\mathrm{P} \geq 10 \mathrm{MVA}$ & $-0,15 \mathrm{Sn} \leq \mathrm{Qn} \leq 0,45 \mathrm{Sn}$ \\
\hline
\end{tabular}

La puissance réactive réellement fournie par le producteur dans cette limite est déterminée par le distributeur, en fonction des impératifs d'exploitation du réseau auquel est raccordée l'installation.

Si l'installation de production comporte des génératrices synchrones, la fourniture de puissance réactive sera obtenue 


\begin{tabular}{|c|c|c|c|c|}
\hline Harmoniques & \multicolumn{2}{|c|}{ BASSE TENSION } & \multicolumn{2}{c|}{ MOYENNE TENSION } \\
\hline & Reçus & Emis & Reçus & Emis \\
\hline Pairs & $\%$ & $\%$ & $\%$ & $\%$ \\
\hline 2 & 2 & En attente de norme & 2 & 2 \\
\hline 4 & 1 & & 1 & 1 \\
\hline 6 à 24 & 0,5 & & 0,5 & 0,5 \\
\hline Impairs & $\%$ & $\%$ & $\%$ & $\%$ \\
\hline 3 & 5 & En attente de norme & 5 & 5 \\
\hline 5 & 6 & & 6 & 5 \\
\hline 7 & 5 & & 5 & 2 \\
\hline 9 & 1,5 & & 1,5 & 3 \\
\hline 11 & 3,5 & & 3,5 & 3 \\
\hline 13 & 3 & & 3 & 2 \\
\hline 15 et 21 & 0,5 & & 0,5 & 2 \\
\hline 17 & 2 & & 2 & 2 \\
\hline 19 & 1,5 & & 1,5 & 2 \\
\hline 23 et 25 & 1,5 & & 1,5 & \\
\hline
\end{tabular}

Tableau 6: Seuils harmoniques.

uniquement par le réglage du courant d'excitation des machines.

Si l'installation de production comporte des génératrices asynchrones, la fourniture de puissance réactive se fera à l'aide de batteries de condensateurs. Ces batteries pourront être installées directement chez le producteur ou au poste source du distributeur. Dans ce dernier cas, le producteur participera au coût des batteries installées au poste pour une quote-part correspondant à la puissance réactive nécessaire à la compensation de la puissance réactive absorbée par son propre groupe et à la fourniture de la quantité Qn.

Afin d'éviter le risque de surtensions et de fonctionnement en autonome lors d'un découplage du réseau, les génératrices asynchrones ne doivent pas rester isolées sur des condensateurs, sans charge. Les génératrices raccordées sur un départ HTA réservé ou très faiblement chargé peuvent se trouver dans une telle situation lors des déclenchements du disjoncteur du départ. Dans ce cas, il faut limiter la quantité de condensateurs de compensation installés localement et donner la préférence à l'installation de condensateurs au poste source, ainsi on peut installer localement les condensateurs compensant uniquement la puissance réactive appelée par les génératrices, les autres étant situés au poste source.

Aucun condensateur de compensation ne doit être associé localement aux génératrices asynchrones raccordées en BT.
Néanmoins, si ces machines sont destinées à fonctionner, par période, en réseau autonome, il est possible de leur coupler des condensateurs de compensation uniquement pendant ces périodes. Un asservissement doit être installé pour ce faire. Lorsque son installation est découplée, il appartient au producteur de protéger ses installations.

\section{VIII — PROTECTIONS DE DÉCOUPLAGE}

Les protections de découplage reprennent la G.T.E. 2666 d'E.D.F. du 17 mars 1982. Elles ont pour objet :

- de permettre le fonctionnement normal des protections et automatismes installés par le distributeur,

- d'éviter le maintien de réseaux séparés sans défaut, afin d'éviter les faux couplages au moment du recouplage de ces réseaux au réseau du distributeur,

- de déconnecter instantanément les installations de production en cas de défaut survenant pendant le régime spécial d'exploitation instauré lors des travaux sous tension effectués sur le réseau aérien HTA.

Les temps d'action de ces protections doivent être coordonnés avec ceux du plan de protection du distributeur.

8.1 Choix du type de protection de découplage (Tab. 7)

\begin{tabular}{|l|c|c|c|c|c|c|}
\hline \multirow{2}{*}{ Protection (1) } & \multicolumn{5}{|c|}{ RESEAU } & \multicolumn{2}{|c|}{ BT } \\
\cline { 2 - 7 } & \multicolumn{5}{|c|}{ HTA } \\
\cline { 2 - 7 } & $\begin{array}{c}\text { Aérien avec RR et RL } \\
\text { automatique }\end{array}$ & $\begin{array}{c}\text { Mixte avec RL } \\
\text { automatique }\end{array}$ & $\begin{array}{c}\text { Souterrain sans } \\
\text { réenclenchement }\end{array}$ & $\begin{array}{c}\text { Liaison directe } \\
\text { producteur poste source }\end{array}$ & P10kVA & P10kVA \\
\hline Ordinaire & 1,1 & 1,2 & 1,2 & 1,2 & 2,1 & 2,2 \\
\hline $\begin{array}{l}\text { Avec relais de } \\
\text { présence tension } \\
\text { si RR }\end{array}$ & 1,3 & 2,2 & 1,3 & 1,3 & 2,1 & \\
\hline $\begin{array}{l}\text { Avec une liaison } \\
\text { fiable au poste } \\
\text { source }\end{array}$ & 1,4 & 1,3 & 1,4 & 1,4 & 2,1 & 2,2 \\
\hline
\end{tabular}

(1) Avec commutateur de mise en R.S.E. si des travaux sous tension sont prévus pour les types 1.2 et 1.3

Tableau 7 : Choix du type de protection de découplage (Conseillée en gras, déconseillée en italique) 
TYPES DE PROTECTION

\begin{tabular}{|c|c|c|c|c|c|c|}
\hline \multirow{3}{*}{$\begin{array}{l}\text { Alimentation } \\
\text { Type }\end{array}$} & \multicolumn{6}{|c|}{ TYPES DE PROTECTION } \\
\hline & \multicolumn{4}{|c|}{ HT } & \multicolumn{2}{|l|}{ BT } \\
\hline & 1,1 & 1,2 & 1,3 & 1,4 & 2,1 & 2,2 \\
\hline Puissance (kVA) & $\forall \mathrm{P}$ & $\forall \mathrm{P}$ & $\forall P$ & $\mathrm{P} \geq 1000$ & $10<\mathrm{P} \leq 250$ & $\mathrm{P} \leq 10$ \\
\hline \multicolumn{7}{|l|}{ Fonctions à assurer } \\
\hline \multicolumn{7}{|c|}{ Ouverture du disjoncteur de départ HTA sur défaut } \\
\hline $\begin{array}{l}\text { Ne pas alimenter un } \\
\text { défaut à la terre }\end{array}$ & $\begin{array}{l}\text { Relais à maximum de } \\
\text { tension homopolaire } \\
\text { instantané }\end{array}$ & $\begin{array}{l}\text { Relais à maximum de } \\
\text { tension homopolaire } \\
\text { temporisé à } 1 \text { ou } 1,5 \mathrm{~s}\end{array}$ & $\begin{array}{l}\text { Relais à maximum et minimum de } \\
\text { fréquence } 49,5 \mathrm{~Hz}-50,5 \mathrm{~Hz} \text { avec en } \\
\text { secours un relais à maximum de tension } \\
\text { homopolaire temporisé à } 1 \text { ou } 1,5 \mathrm{~s}\end{array}$ & $\begin{array}{l}\text { Asservissement avec en secours maximum } \\
\text { de tension homopolaire instantané et } \\
\text { temporisé à } 1 \text { ou } 1,5 \mathrm{~s} \text { et minimum et } \\
\text { maximum de fréquence } 49,5 \mathrm{~Hz}-50,5 \mathrm{~Hz}\end{array}$ & $\begin{array}{l}3 \text { relais à minimum de } \\
\text { tension } 85 \% \text { instantanés }\end{array}$ & $\begin{array}{c}3 \text { relais à minimum } \\
\text { de tension } 85 \% \\
\text { instantanés }\end{array}$ \\
\hline $\begin{array}{l}\text { Ne pas alimenter un } \\
\text { défaut entre phases }\end{array}$ & $\begin{array}{c}3 \text { relais à minimum de } \\
\text { tension } 85 \% \text { instantanés }\end{array}$ & $\begin{array}{l}3 \text { relais à minimum de } \\
\text { tension } 85 \% \text { instantanés }\end{array}$ & $\begin{array}{c}\text { Relais à maximum et minimum de } \\
\text { fréquence } 49,5 \mathrm{~Hz}-50,5 \mathrm{~Hz} \text { avec en } \\
\text { secours } 3 \text { relais à minimum de tension } \\
85 \% \text { temporisé à l ou } 1,5 \mathrm{~s}\end{array}$ & $\begin{array}{c}\text { Asservissement avec en secours } 3 \text { relais à } \\
\text { minimum de tension } 85 \% \\
\text { temporisé à } 1 \text { ou } 1,5 \mathrm{~s}\end{array}$ & $\begin{array}{l}3 \text { relais à minimum de } \\
\text { tension } 85 \% \text { instantanés }\end{array}$ & $\begin{array}{c}3 \text { relais à minimum } \\
\text { de tension } 85 \% \\
\text { instantanés }\end{array}$ \\
\hline $\begin{array}{l}\text { Ne pas enclencher sur } \\
\text { une installation non } \\
\text { découplée }\end{array}$ & $\begin{array}{l}\text { Sans objet } \\
\text { (découplage } \\
\text { instantané) }\end{array}$ & $\begin{array}{c}\text { Verrouillage au poste } \\
\text { source sur présence de } \\
\text { tension }\end{array}$ & $\begin{array}{l}\text { Verrouillage du réenclenchement } \\
\text { rapide au poste source sur présence } \\
\text { de tension }\end{array}$ & $\begin{array}{c}\text { Sans objet } \\
\text { (découplage instantané) }\end{array}$ & $\begin{array}{c}\text { Sans objet } \\
\text { (découplage instantané) }\end{array}$ & $\begin{array}{l}\text { Sans objet } \\
\text { (découplage } \\
\text { instantané) }\end{array}$ \\
\hline \multicolumn{7}{|c|}{ Ouverture du disjoncteur de départ HTA sans qu'il y ait un défaut sur ce départ } \\
\hline $\begin{array}{l}\text { Ne pas alimenter les } \\
\text { autres clients avec } \\
\text { une tension ou une } \\
\text { fréquence anormale }\end{array}$ & \begin{tabular}{|c|} 
Relais à maximum et \\
minimum de fréquence \\
$47,5 \mathrm{~Hz}-51 \mathrm{~Hz}$ et \\
minimum et maximum de \\
tension $85 \%$ et $115 \%$ \\
instantanés \\
\end{tabular} & \begin{tabular}{|c|} 
Relais à maximum et \\
minimum de fréquence \\
$47,5 \mathrm{~Hz}-51 \mathrm{~Hz}$ et \\
minimum et maximum de \\
tension $85 \%$ et $115 \%$ \\
instantanés \\
\end{tabular} & $\begin{array}{l}\text { Relais à maximum et minimum de } \\
\text { fréquence } 51 \mathrm{~Hz}-47,5 \mathrm{~Hz} \text { instantané et } \\
\text { minimum } 25 \% \text { de tension instantané et } \\
\text { minimum de tension } 85 \% \text { temporisé et } \\
\text { maximum de tension } 115 \% \text { instantanés }\end{array}$ & $\begin{array}{l}\text { Asservissement et, relais à maximum et } \\
\text { minimum de fréquence } 51 \mathrm{~Hz}-47,5 \mathrm{~Hz} \\
\text { instantané et minimum } 25 \% \text { de tension } \\
\text { instantané et minimum de tension } 85 \% \\
\text { temporisé et maximum de tension } 115 \% \\
\text { instantanés }\end{array}$ & $\begin{array}{l}3 \text { relais à minimum de } \\
\text { tension } 85 \% \text { instantanés et } \\
\text { un maximum de tension } \\
110 \% \text { instantané et } \\
\text { minimum et maximum de } \\
\text { fréquence } 49,5 \mathrm{~Hz}-50,5 \mathrm{~Hz} \\
\end{array}$ & $\begin{array}{c}3 \text { relais à minimum } \\
\text { de tension } 85 \% \\
\text { instantanés et un } \\
\text { maximum de } \\
\text { tension } 110 \% \\
\text { instantané } \\
\end{array}$ \\
\hline $\begin{array}{l}\text { Ne pas enclencher sur } \\
\text { une installation non } \\
\text { découplée }\end{array}$ & $\begin{array}{l}\text { Relais à minimum de } \\
\text { tension } 85 \% \text { instantanés }\end{array}$ & $\begin{array}{l}\text { Relais à minimum de } \\
\text { tension } 85 \% \text { instantanés }\end{array}$ & $\begin{array}{l}\text { Relais à minimum de tension } 25 \% \\
\text { instantanés }\end{array}$ & $\begin{array}{l}\text { Relais à minimum de tension } 25 \% \\
\text { instantanés }\end{array}$ & $\begin{array}{l}3 \text { relais à minimum de } \\
\text { tension } 85 \% \text { instantanés }\end{array}$ & $\begin{array}{c}3 \text { relais à minimum } \\
\text { de tension } 85 \% \\
\text { instantanés }\end{array}$ \\
\hline \multicolumn{7}{|c|}{ Défauts sur d'autres départs HTA } \\
\hline $\begin{array}{l}\text { En cas de baisse de } \\
\text { tension }<25 \% \\
\text { déphasage sur un } \\
\text { alternateur non } \\
\text { découplé }\end{array}$ & $\begin{array}{c}\text { Relais à minimum de } \\
\text { tension } 85 \% \text { instantanés }\end{array}$ & $\begin{array}{l}\text { Relais à minimum de } \\
\text { tension } 85 \% \text { instantanés }\end{array}$ & $\begin{array}{l}\text { Relais à minimum de tension } 25 \% \\
\text { instantanés }\end{array}$ & $\begin{array}{l}\text { Relais à minimum de tension } 25 \% \\
\text { instantanés }\end{array}$ & $\begin{array}{l}3 \text { relais à minimum de } \\
\text { tension } 85 \% \text { instantanés }\end{array}$ & $\begin{array}{c}3 \text { relais à minimum } \\
\text { de tension } 85 \% \\
\text { instantanés }\end{array}$ \\
\hline \multicolumn{7}{|c|}{ Défaut sur alimentation HTB } \\
\hline \multicolumn{7}{|c|}{ Travaux sous tension prévisibles sur le départ HTA } \\
\hline $\begin{array}{l}\text { Mise en régime } \\
\text { spécial d'exploitation } \\
\text { mise à } 0 \text { des tempos }\end{array}$ & $\begin{array}{c}\text { Sans objet } \\
\text { (découplage } \\
\text { instantané) }\end{array}$ & $\begin{array}{l}\text { Commutateur de mise } \\
\text { en R.S.E. }\end{array}$ & Commutateur de mise en R.S.E. & $\begin{array}{c}\text { Sans objet } \\
\text { (découplage instantané) }\end{array}$ & $\begin{array}{c}\text { Sans objet } \\
\text { (découplage instantané) }\end{array}$ & $\begin{array}{c}\text { Sans objet } \\
\text { (découplage } \\
\text { instantané) }\end{array}$ \\
\hline
\end{tabular}




\begin{tabular}{|c|c|c|c|c|}
\hline \multirow{2}{*}{$\frac{\text { Raccordement }}{\text { Comptage }}$} & \multicolumn{2}{|c|}{ Basse tension } & \multicolumn{2}{|c|}{ Haute tension } \\
\hline & $\begin{array}{l}\text { Basse tension } \\
\text { avec } P \quad 36 \text { kVA }\end{array}$ & $\begin{array}{c}\text { Basse tension } \\
\text { avec } 36 \mathrm{P}(\mathrm{kVA}) \\
250\end{array}$ & $\begin{array}{c}\text { Basse tension } \\
\text { possible si } 1 \text { transfo } \\
\text { et rapport des TC } \\
2000 / 5\end{array}$ & Haute tension \\
\hline TC & $\mathrm{Cl} 0,5$ & $\mathrm{Cl} 0,5$ & $\mathrm{Cl} 0,5$ & $\mathrm{Cl} \mathrm{0,5}$ \\
\hline TP & Sans objet & Sans objet & Sans objet & Monophasé $\mathrm{Cl} 0,5$ \\
\hline Compteurs & Type bleu & Type jaune & Type vert & $\begin{array}{l}\text { Type vert dans } \\
\text { les } 2 \text { sens }\end{array}$ \\
\hline Lecture & $\begin{array}{l}\text { Télérelevable } \\
\text { localement }\end{array}$ & $\begin{array}{l}\text { Télérelevable } \\
\text { localement }\end{array}$ & $\begin{array}{l}\text { Télérelevable } \\
\text { à distance }\end{array}$ & $\begin{array}{l}\text { Télérelevable } \\
\text { à distance }\end{array}$ \\
\hline $\begin{array}{l}\text { Qualité de } \\
\text { fourniture }\end{array}$ & & & & $\begin{array}{l}1 \text { indicateur } \\
\text { de coupures }\end{array}$ \\
\hline autoconsommateur & & & & $\begin{array}{l}1 \text { comptage } \\
\text { type client }\end{array}$ \\
\hline
\end{tabular}

Tableau 9: Les différents comptages

\subsection{Principes de détection}

Les principes de détections suivants sont utilisés.

Un relais à maximum de tension homopolaire pour les défauts H.T.A. à la terre. Trois relais à minimum de tension réglés à $85 \%$ pour la détection des défauts entre phases. Un relais à maximum de tension réglé à $115 \%$, un relais à minimum et maximum de fréquence réglé à 47,5 et $51 \mathrm{~Hz}$ et les relais à minimum de tension réglés à $85 \%$ pour la détection du réseau séparé sans défaut.

\subsection{Types de protections}

( Voir tableau 8)

\section{MISES À LA TERRE DES MASSES ET DU NEUTRE}

\subsection{Raccordement en basse tension}

De manière générale, le neutre du réseau BT ne doit pas être relié à la terre dans l'installation du producteur tant qu'elle est raccordée au réseau. Si elle doit l'être en fonctionnant en réseau séparé, un asservissement doit être installé entre la mise à la terre du neutre et le couplage.

Toutefois, si le réseau électrique le permet (c'est-à-dire si le réseau et la terre de l'installation de production à raccorder respectent les mêmes conditions que celles qui sont requises pour le réseau et la terre du client dans le cadre d'un contrat de fourniture d'électricité au tarif jaune), la connexion du neutre du réseau BT à la terre dans l'installation du producteur est possible à la demande du producteur, après accord du distributeur.

\subsection{Raccordement en HTA}

Les écrans du câble souterrain desservant éventuellement l'installation sont reliés entre eux et doivent être reliés aux masses de ce poste.

Si l'installation est contiguë au poste source, il est préférable d'interconnecter les masses de l'installation et celles du poste source. Les conducteurs d'interconnexion doivent être dimensionnés en fonction de la valeur des courants de défauts qui peuvent les parcourir.

Le neutre HTA ne doit pas être relié à la terre au poste de l'installation du producteur lorsque cette installation est raccordée au réseau.

\section{$X$ TRANSMISSION DES ORDRES EJP}

Elle peut se faire par la voie ordinaire, à l'aide du signal habituel $175 \mathrm{~Hz}$ envoyé à tous les clients sur le réseau.

\section{XI $\square$ COMPTAGES}

Les matériels de comptages sont conformes aux normes de la famille NF C44000.

Les comptages sont réalisés conformément à la norme NF C14-100 en BT et NF C13-100 en HT. 